\title{
Formation and migration of helium pair in bcc Fe from first principle calculations
}

\author{
Liuliu Li ${ }^{\mathrm{a}}$, Jingyi Shi ${ }^{\mathrm{b}}$, Lei Peng ${ }^{\mathrm{a}, *}$, Wei Jiang ${ }^{\mathrm{a}}$, Guian Qian ${ }^{\mathrm{c}}$ \\ ${ }^{\text {a }}$ School of Physical Sciences, University of Science and Technology of China, Hefei, Anhui 230027, China \\ ${ }^{\mathrm{b}}$ Advanced Energy Research Center, Shenzhen University, Shenzhen 518060, China \\ ${ }^{\mathrm{c}}$ State Key Laboratory for Nonlinear Mechanics, Institute of Mechanics, Chinese Academy of Sciences, Beijing 100190, China
}

\section{A R T I C L E I N F O}

\section{Keywords:}

First-principle

Helium pair

Migration

Bcc Fe

\begin{abstract}
A B S T R A C T
We investigated the energetics and configurations of two interstitial He atoms in bcc Fe crystal using firstprinciple method. Two interstitial He atoms will bind together as helium pair if the initial He-He distance is less than $2.82 \AA$ A. From a formation energy decomposing analysis we found that, pairing of two He atoms would reduce the interface between $\mathrm{He}$ and Fe matrix which mitigates the perturbation of He-1s and Fe-3d orbitals. As a dominant part of the fomation energy, the electron variation energy caused by two close He atoms is less than that of two far He atoms, which accounts for the reason of binding between two close He atoms. Besides, the migration of helium pair along direction $\left[\begin{array}{lll}1 & 0 & 0\end{array}\right]$ was investigated by a He-He formation energy hypersurface. Through the comparison with Nudged Elastic Band calculation, it's found that this special energy hypersurface was effective in revealing the migration of helium pair in metals.
\end{abstract}

\section{Introduction}

It's well known that a large quantity of helium atoms are introduced in fusion structure materials by $(n, \alpha)$ transmutation reaction. The helium can be deeply trapped in lattice defects such as vacancy, void, dislocation and grain boundary due to its low solubility and high mobility in metals and grows into helium bubble, degrading the mechanical properties of the materials [1-3]. Revealing the He-He and Hemetal interactions is imperative for the development of fusion structure materials.

In metallic alloys, helium atoms produced by nuclear reaction would firstly form at interstitial sites $[4,5]$ and initially accumulate into small interstitial helium clusters [6-8]. G. Thomas et al. [9] found that a large proportion of helium will stay in metals, which is probably the consequence of He-He interaction. It was also proved that dislocation loops and helium bubbles would form in defect-free lattice [10], which is the evidence for spontaneous precipitation of helium in metals. Besides, helium atoms was found to cluster at the near-surface region in tungsten [11-13], which was later attributed to the interaction between helium atoms. An atomistic insight into helium trapping in metals is needed. However, it's difficult to determine the atomistic properties of helium atoms in metals experimentally. To explain the experiment findings from computer simulation, Wilson et al. [14] studied the binding of helium atoms in metals by atomistic calculations. It was found that two interstitial helium atoms have large binding energy, called helium self-trapping. Then, a rate theory simulation which is based on the binding energy between two interstitial He atoms was applied to analyze the evolution of helium clusters below room temperature [15]. Later, a multi-scale approach was adopted and the results showed that the near-surface clustering of helium in $\mathrm{W}$ was originated from the bound state between helium atoms [16].

An in-depth study of the binding property of two near interstitial He atoms in metal, i.e., helium pair [16-19], is a fundamental starting point for the understanding of helium self-trapping behavior. It is important to investigate the complex multi ion-electron interaction, which could be accurately described by the first-principle simulation. And the first application is for single helium atom behavior in bcc Fe $[20,21]$. Then Domain et al. [22] studied the binding property of twointerstitial He atoms in tungsten by ab initio calculations. Later, the migration of helium pair in metals was intensively studied using the nudged elastic band (NEB) method $[17,19]$. It was also found that large binding energy between two He atoms is common in transition metals $[18,19]$. As a closed-shell noble gas atom, it's strange for one He atom to bind with another. To find the origin of He-He binding, Zhang et al. [18] calculated the density of state of He-He in metals and found that the density of state (DOS) value at Fermi level of helium pair is lower than that of two far interstitial He atoms in group VB and VIB transition metals. Generally, higher value of DOS at Fermi level means less

\footnotetext{
* Corresponding author.

E-mail address: penglei@ustc.edu.cn (L. Peng).
} 
energetically favorable $[18,23,24]$. So He-He pair would be more stable than two separated $\mathrm{He}$ atoms. Wang et al. [25] found that $\mathrm{He}-\mathrm{He}$ binding in metals is related not only to the electron density variation but also to the host lattice distortion.

In this study, we systematically investigated the configurations and energetics of interstitial helium pairs in bcc Fe crystal by ab initio calculations. We revealed the reason of $\mathrm{He}-\mathrm{He}$ binding in bcc $\mathrm{Fe}$ through energy decomposing analysis. We also constructed a He-He formation energy hypersurface and efficiently demonstrated the helium pair migration in bcc Fe bulk.

\section{Methodology}

Calculations were performed using spin polarized density functional theory (DFT) and plane-wave pseudopotential approach [26,27], as implemented in the Vienna Ab initio Simulation Package (VASP) [28]. We described the electron-ion interaction with projector augmented wave (PAW) potentials $[29,30]$ and the exchange-correlation interaction with generalized gradient approximation (GGA) in the Perdew Burke Ernzerhof (PBE) form [30]. The bcc supercell contained 128 atoms ( $4 \times 4 \times 4$ unit cells) and the Brillouin zones were sampled by Monkhorst-Pack scheme [31]. After performing a series of parameter optimizations, we chose a k-points mesh of $3 \times 3 \times 3$, a plane wave cut-off energy of $450 \mathrm{eV}$, and a Methfessel-Paxton smearing width of $0.02 \mathrm{eV}$. The energy minimization was converged until the energy difference was less than $1.0 \times 10^{-5} \mathrm{eV}$ and the forces on all the atoms were less than $0.005 \mathrm{eV} / \AA$. The zero point energies (ZPE) of tetrahedral interstitial sites (TIS) and octahedral interstitial site (OIS) He atoms are $0.076 \mathrm{eV}$ and $0.030 \mathrm{eV}$, similar to the values of $0.072 \mathrm{eV}$ and $0.031 \mathrm{eV}$ in Ref. [32]. ZPE correction was not considered in this work due to the small value. In the migration calculation, we used 54 atoms $(3 \times 3 \times 3$ unit cells) supercell, which is sufficient for the calculation of the energy barrier as indicated in Table 1. For the migration calculation with Nudged Elastic Band (NEB) [33] method, three images were linearly interpolated between each pair of neighboring local energy minima on the migration track and the volume of the supercell as well as the atomic positions were relaxed. The migration energies were determined from the saddle point of the energy profile.

The formation energy and binding energy of the N Fe-M He system were calculated as follows:

$E_{\mathrm{Fe}_{\mathrm{N}} H e_{\mathrm{M}}}^{f}=E_{\mathrm{Fe}_{\mathrm{N}} H e_{\mathrm{M}}}-E_{\mathrm{Fe}_{\mathrm{N}} \text { perfect }}-\mathrm{M} E_{\mathrm{He}}$

where $E_{\mathrm{Fe}_{\mathrm{N}} H e_{\mathrm{M}}}^{f}$ is the formation energy of the system containing $\mathrm{N} \mathrm{Fe}$ atoms and $\mathrm{M} \mathrm{He}$ atoms. $E_{\mathrm{Fe}_{\mathrm{N}} \mathrm{He}_{\mathrm{M}}}$ is the energy of the relaxed system. $E_{F_{\mathrm{N}} \text { perfect }}$ is the energy of a perfect bcc Fe crystal containing N Fe atoms. $E_{H e}$ is the energy of an isolated He atom. The values of formation energy reflects the difficulty of the formation of the system; larger value means more unstable.

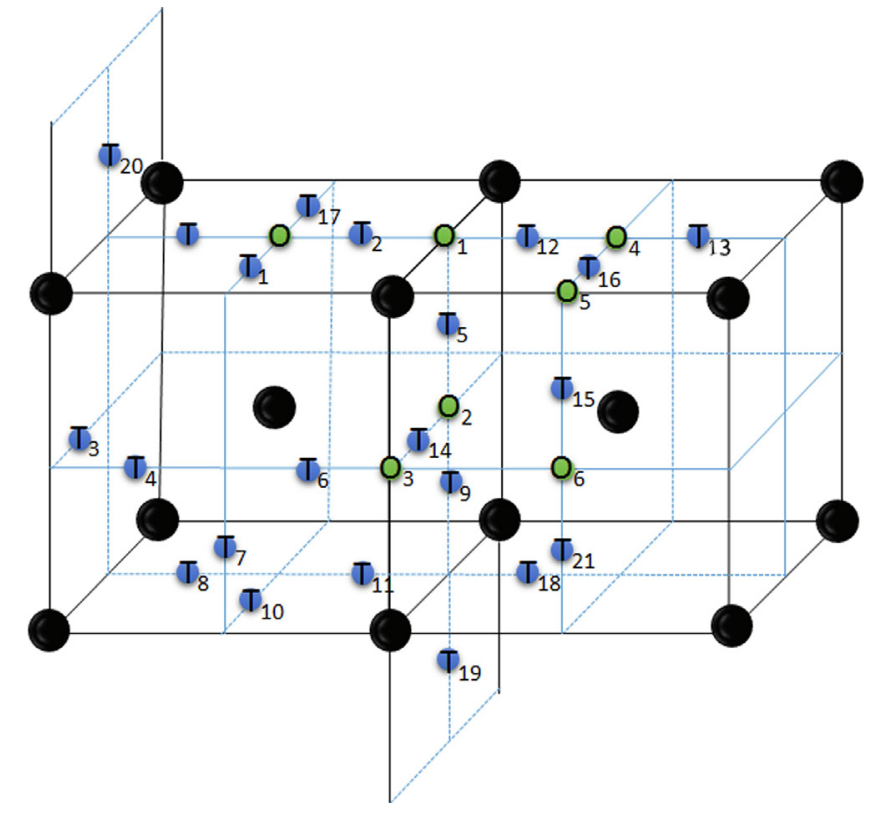

Fig. 1. The initial positions of He-He configurations. Black spheres refer to Fe atoms. Blue and green spheres refer to O (OIS) and T (TIS), which are labelled accordingly with subscripts. Configurations $\mathrm{TT}_{1} / \mathrm{OO}_{1}, \mathrm{TT}_{2} / \mathrm{OO}_{2} \ldots \mathrm{TT}_{6} / \mathrm{OO}_{6}$ refer to the first, second...sixth nearest neighbor TIS-TIS/OIS-OIS configurations. (For interpretation of the references to color in this figure legend, the reader is referred to the web version of this article.)

$E_{\mathrm{Fe}_{\mathrm{N}} H e_{2}}^{b}=2 E_{\mathrm{Fe}_{\mathrm{N}} \mathrm{He} e_{\mathrm{T}}}^{f}-E_{\mathrm{Fe}_{\mathrm{N}} \mathrm{He}}^{f}$

where $E_{\mathrm{Fe}_{\mathrm{N}} \mathrm{He}_{2}}^{b}$ and $E_{\mathrm{Fe}_{\mathrm{N}} \mathrm{He}_{2}}^{f}$ are the binding and formation energy of two interstitial He atoms in N Fe crystal. $E_{\mathrm{Fe}_{\mathrm{N}} \mathrm{HeT}}^{f}$ is the formation energy of the most stable single interstitial He atoms in N Fe bulk where He atom locates at tetrahedral site. Larger binding energy corresponds to stronger attraction, while negative values mean repulsive interaction.

The calculated equilibrium lattice constant $a$, cohesive energy of bcc Fe atom $\left(E_{c o h}\right)$, vacancy formation energy $\left(E_{v}^{f}\right)$, substitutional helium formation energy $\left(E_{S u b}^{f}\right)$, TIS helium formation energy $\left(E_{T I S}^{f}\right)$, and OIS helium formation energy $\left(E_{O I S}^{f}\right)$, as well as the He atom migration energy are listed in Table 1.

\section{Results and discussion}

\subsection{Formation of helium pair}

As illustrated in Fig. 1, we calculated 27 initial He-He configurations, including the first to sixth nearest neighbor OIS-OIS, TIS-TIS and

Table 1

Properties of Fe crystal and Fe-He system in present work and some references.

\begin{tabular}{|c|c|c|c|c|c|c|c|c|}
\hline & Supercell & $a(\AA)$ & $E_{c o h}(\mathrm{eV})$ & $E_{v}^{f}(\mathrm{eV})$ & $E_{S u b}^{f}(\mathrm{eV})$ & $E_{T I S}^{f}(\mathrm{eV})$ & $E_{O I S}^{f}(\mathrm{eV})$ & $E_{H e}^{m}(\mathrm{eV})$ \\
\hline \multirow[t]{2}{*}{ Present } & $3 \times 3 \times 3$ & 2.87 & 5.05 & 2.16 & 4.29 & 4.45 & 4.64 & 0.06 \\
\hline & $4 \times 4 \times 4$ & 2.82 & 5.28 & 2.16 & 4.36 & 4.64 & 4.86 & \\
\hline \multirow[t]{4}{*}{ Reference } & $4 \times 4 \times 4$ & $2.82^{\mathrm{a}}$ & $5.28^{\mathrm{a}}$ & $2.16^{\mathrm{a}}$ & & $4.65^{\mathrm{a}}$ & $4.85^{\mathrm{a}}$ & $0.06^{\mathrm{a}}$ \\
\hline & & $2.83^{\mathrm{b}}$ & $5.26^{\mathrm{b}}$ & $2.14^{\mathrm{b}}$ & $4.34^{\mathrm{b}}$ & $4.56^{\mathrm{b}}$ & $4.75^{\mathrm{b}}$ & $0.06^{\mathrm{f}}$ \\
\hline & & $2.82^{\mathrm{c}}$ & $5.10^{c}$ & $2.18^{\mathrm{e}}$ & $4.37^{\mathrm{e}}$ & $4.59^{\mathrm{e}}$ & $4.81^{\mathrm{e}}$ & $0.06^{g}$ \\
\hline & & $2.83^{\mathrm{d}}$ & & $2.14^{\mathrm{d}}$ & $4.35^{\mathrm{d}}$ & $4.59^{\mathrm{d}}$ & & \\
\hline
\end{tabular}

\footnotetext{
a Ref. [34].

b Ref. [24].

c Ref. [18].

d Ref.[35]

e Ref. [36].

f Ref. [20].

g Ref. [37].
} 
Table 2

Initial and final He-He distances, He atom occupancy sites, as well as the formation and binding energies of the He-He configurations.

\begin{tabular}{|c|c|c|c|c|c|c|c|}
\hline \multicolumn{3}{|c|}{ Initial He-He configurations } & \multicolumn{5}{|c|}{ Optimized He-He configurations } \\
\hline Name & Sites & Distance/Å & Name & Sites & Distance/Å & Formation energy/eV & Binding Energy/eV \\
\hline I1 & $\mathrm{OT}_{5}$ & 1.58 & $\mathrm{~F} 1$ & $\mathrm{TT}_{5}$ & 1.60 & 8.79 & 0.49 \\
\hline I2 & $\mathrm{TT}_{5}$ & 2.23 & & $\mathrm{TT}_{5}$ & & & \\
\hline I3 & $\mathrm{OT}_{9}$ & 2.55 & & $\mathrm{~T}_{2} \mathrm{~T}_{9}$ & & & \\
\hline I4 & $\mathrm{TT}_{3}$ & 1.73 & F2 & $\mathrm{TT}_{3}$ & 1.63 & 8.81 & 0.47 \\
\hline I5 & $\mathrm{OT}_{14}$ & 2.12 & & $\mathrm{~T}_{2} \mathrm{~T}_{14}$ & & & \\
\hline I6 & $\mathrm{TT}_{7}$ & 2.64 & & $\mathrm{~T}_{1} \mathrm{~T}_{4}$ & & & \\
\hline I7 & $\mathrm{TT}_{6}$ & 2.45 & F3 & $\mathrm{TT}_{6}$ & 1.61 & 8.86 & 0.42 \\
\hline I8 & $\mathrm{OT}_{2}$ & 0.71 & F4 & $\mathrm{TT}_{2}$ & 1.58 & 8.89 & 0.39 \\
\hline I9 & $\mathrm{TT}_{2}$ & 1.41 & & $\mathrm{TT}_{2}$ & & & \\
\hline I10 & $\mathrm{OT}_{12}$ & 2.12 & & $\mathrm{~T}_{2} \mathrm{~T}_{12}$ & & & \\
\hline I11 & $\mathrm{OO}_{4}$ & 2.82 & & $\mathrm{~T}_{2} \mathrm{~T}_{12}$ & & & \\
\hline I12 & $\mathrm{TT}_{4}$ & 2.00 & F5 & $\mathrm{TT}_{4}$ & 1.66 & 8.92 & 0.36 \\
\hline I13 & $\mathrm{OO}_{3}$ & 2.45 & F6 & $\mathrm{T}_{1} \mathrm{~T}_{6}$ & 1.61 & 8.96 & 0.32 \\
\hline I14 & $\mathrm{TT}_{1}$ & 1.00 & F7 & $\mathrm{TT}_{1}$ & 1.53 & 9.04 & 0.24 \\
\hline I15 & $\mathrm{OO}_{2}$ & 2.00 & & $\mathrm{~T}_{2} \mathrm{~T}_{5}$ & & & \\
\hline I16 & $\mathrm{OO}_{1}$ & 1.41 & F8 & $\mathrm{OO}_{1}$ & 1.68 & 9.12 & 0.15 \\
\hline I17 & $\mathrm{OT}_{16}$ & 2.91 & F9 & $\mathrm{T}_{17} \mathrm{~T}_{16}$ & 3.06 & 9.14 & 0.14 \\
\hline I18 & $\mathrm{OO}_{5}$ & 3.16 & & $\mathrm{~T}_{17} \mathrm{~T}_{16}$ & & & \\
\hline I19 & $\mathrm{TT}_{12}$ & 2.82 & F10 & $\mathrm{TT}_{12}$ & 2.76 & 9.16 & 0.12 \\
\hline I 20 & $\mathrm{OT}_{11}$ & 2.91 & F11 & $\mathrm{T}_{2} \mathrm{~T}_{19}$ & 3.60 & 9.21 & 0.07 \\
\hline I21 & $\mathrm{OT}_{15}$ & 3.24 & F12 & $\mathrm{T}_{17} \mathrm{~T}_{15}$ & 3.60 & 9.22 & 0.06 \\
\hline I22 & $\mathrm{OO}_{6}$ & 3.46 & F13 & $\mathrm{T}_{17} \mathrm{~T}_{21}$ & 4.09 & 9.24 & 0.04 \\
\hline I23 & $\mathrm{TT}_{13}$ & 4.21 & F14 & $\mathrm{TT}_{13}$ & 4.24 & 9.24 & 0.03 \\
\hline I 24 & $\mathrm{TT}_{10}$ & 3.00 & F15 & $\mathrm{TT}_{10}$ & 5.05 & 9.24 & 0.03 \\
\hline I25 & $\mathrm{TT}_{11}$ & 3.16 & F16 & $\mathrm{T}_{20} \mathrm{~T}_{19}$ & 5.16 & 9.25 & 0.03 \\
\hline I26 & $\mathrm{TT}_{8}$ & 2.82 & F17 & $\mathrm{TT}_{8}$ & 2.90 & 9.31 & -0.03 \\
\hline I27 & $\mathrm{TT}_{9}$ & 3.00 & F18 & $\mathrm{T}_{20} \mathrm{~T}_{18}$ & 3.43 & 9.39 & -0.11 \\
\hline
\end{tabular}

OIS-TIS configurations as well as several other configurations with larger He-He distances. The initial and optimized distances and the formation energies are listed in Table 2. The initial configurations are named as I1-I27. After optimization, there are 18 different final configurations named as F1-F18, following the order of the formation energies.

Apparently in Fig. 2, the He-He configurations could be divided into two groups according to the initial He-He distances. One group contains the configurations F1-F8: the initial He-He distances are below $2.82 \AA$ and the final distances are of 1.5-1.7 $\AA$, and the binding energies are large. Namely, this kind of He-He configurations is called helium pair. The other contains the configurations F9-F18: the initial He-He distances are above $2.82 \AA$ and the final distances are generally enlarged. This kind of He-He configurations with small or negative binding energies is called far He-He, indicating little binding interaction between the two He atoms.

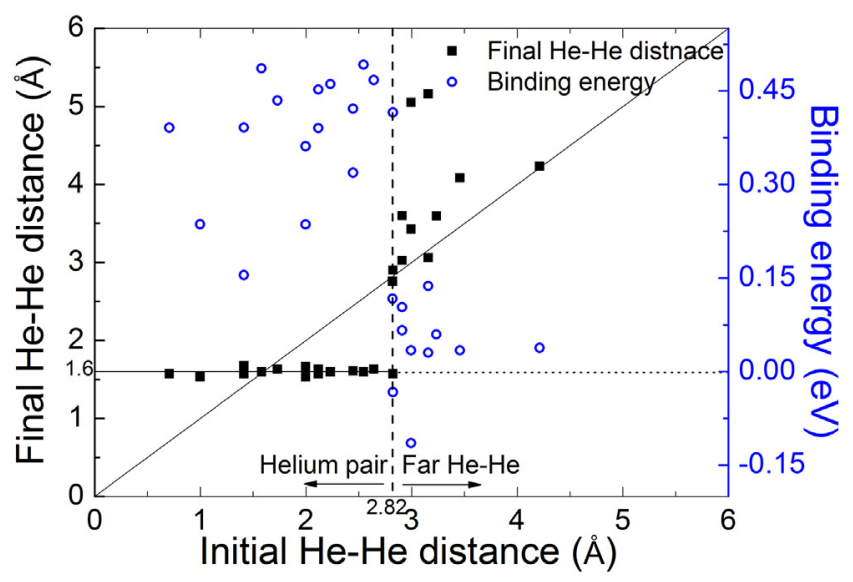

Fig. 2. Initial/optimized He-He distances and binding energies of He-He configurations. Lines corresponding to initial He-He distance $2.82 \AA$, final distance $1.6 \AA$ and binding energy $0 \mathrm{eV}$ are also plotted to guide the eye.

\subsection{Energy decomposing analysis}

In a recent report, it was suggested that binding between two $\mathrm{He}$ atoms is related to both electron variation and lattice distortion [25]. On the basis of charge density difference equation, we put forward a formula to decompose $\mathrm{He}-\mathrm{He}$ formation energy into electron part and lattice part. Similar formation energy decomposing method could be found in Refs. [30-32].

The equation of charge density difference for the N Fe-M He supercell:

$\Delta \rho=\rho_{\mathrm{Fe}_{\mathrm{N}} \mathrm{He}_{\mathrm{M}}}-\rho_{\mathrm{Fe}_{\mathrm{M}}}-\rho_{\mathrm{He} \mathrm{M}}$

We calculate the energy of each term in Eq. (3):

$\Delta E=E_{\mathrm{Fe}_{\mathrm{N}} \mathrm{He}_{\mathrm{M}}}-E_{\mathrm{Fe}_{\mathrm{N}}}-E_{\mathrm{He} \mathrm{M}}$

where $\Delta \rho$ is the charge density difference and $\Delta E$ is the energy of charge density difference induced by electron density variation. We rewrite $\Delta E$ as $E_{\text {electron }}$ and also rewrite $E_{F e_{\mathrm{N}}}$ as $E_{\mathrm{Fe}_{\mathrm{N}} \text { distort }}$, distinguished from $E_{\text {Fe }_{\text {_perfect }}}$.

If we define:

$E_{\text {lattice }}=E_{F_{e_{\mathrm{N}} \_ \text {distort }}}-E_{\mathrm{Fe}_{\mathrm{N} \_ \text {perfect }}}$

$E_{\text {He_vacuum }}^{b}=\mathrm{M} E_{H e}-E_{H e \mathrm{M}}$

Then the formation energy of the system could be expressed as:

$E_{\mathrm{Fe}_{\mathrm{M}} \mathrm{He} \mathrm{M}}^{f}=E_{\text {electron }}+E_{\text {lattice }}-E_{\text {He_vacuum }}^{b}$

where $E_{\text {electron }}$ refers to the energy of electron part corresponding to the Fe-3d and He-1s orbital interaction [38]. Elattice_distort is the energy of lattice part which arises from the displacements of Fe atoms from their origin lattice sites. $E_{\text {He_vacuum }}^{b}$ is the binding energy of two He atoms in vacuum. Therefore, the formation energy is decomposed into electron variation part, lattice distortion part and an extra part of He-He binding in vacuum.

We decomposed the formation energies of the configurations F1-F18 as shown in Fig. 3. The corresponding energy parts of two independent 


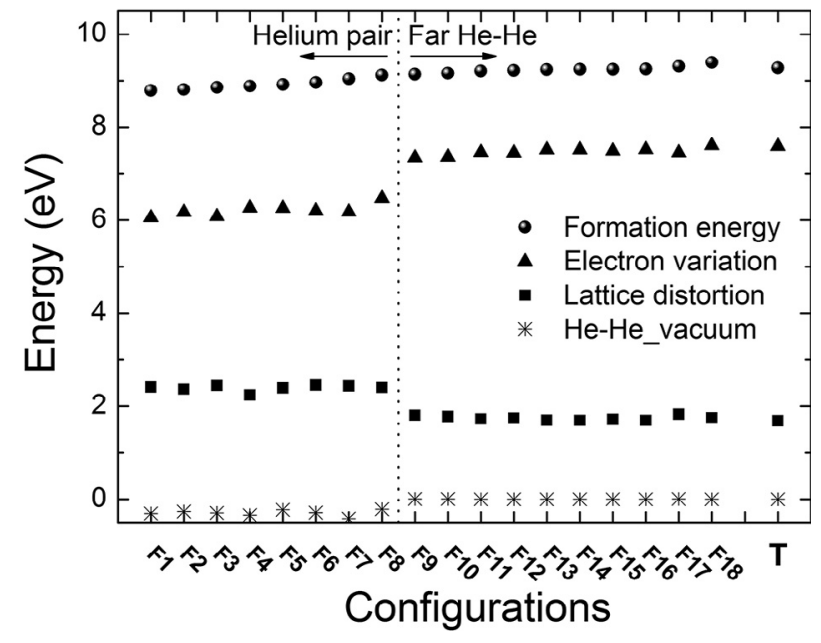

Fig. 3. Energy decomposing analysis of F1-F18. The dotted line divides helium pairs and far He-He. The energy parts of two independent T-site helium atoms are shown, labelled as $\mathrm{T}$ in abscissa.

TIS helium atoms are also shown as a comparison. The binding energy of He-He in vacuum is generally negative, especially when the two He atoms are close, confirming that He atoms actually repel each other. However, this repulsive energy of He-He in vacuum is negligible compared to the electron variation energy or lattice distortion energy. It shows that the electron variation energy is the dominant part in formation energy, while the lattice distortion value is relative small. This result suggests that the electronic interaction between the He and host atoms is important in deciding helium behavior at microscopic level, similar to the results in Refs. $[18,21,23]$. It is interesting that the electron variation energies of helium pairs are smaller than those of far He-He while the lattice distortion energies are just the reverse. This result could be reasonable understood. From Fig. 4 we can see that the $1 \mathrm{~s}$ electron clouds of He atoms strongly interact with the $3 \mathrm{~d}$ electron clouds of nearby Fe atoms, in consistent with $[38,39]$. By analyzing the configurations of the optimized He-He, it was found that two or three $\mathrm{Fe}$ atoms at the tetrahedral vertexes are shared by the two TIS He atoms in helium pair; while for far $\mathrm{He}-\mathrm{He}$, one or none vertex Fe atom are shared by the two $\mathrm{He}$ atoms. In other words, the $\mathrm{He} / \mathrm{Fe}$ interface is less for helium pair compared with far He-He. As a result, the two He atoms in helium pairs cause less Fe-3d and He-1s electron cloud electron variation; and the Fe atoms at the shared vertex feel stronger Fe-He repulsion than other $\mathrm{Fe}$ atoms which causes larger lattice distortion. As electron density variation is dominant in formation energy, two He atoms tend to form helium pair rather than far He-He. Besides, the energy parts of far He-He are comparable with those of two independent TIS helium atoms, implying that far He-He with distance above $2.82 \AA$ are similar to two independent He atoms.

\subsection{Helium pair migration along direction [1 00 ]}

A formation energy hypersurface was applied to analyze the migration of helium pairs in bcc Fe. Here, we start with a simple condition where two He atoms are restrained to move along direction [1 000 , i.e. line L shown in Fig. 5 (a). The $x-y$ coordinate plane is shown in the Fig. 5 (b). The three dimensional overview in shown in Fig. 5(c). The projection of the hypersurface on the x-y plane is shown in Fig. 5(d). In Fig. 5 (d) we can see that, for final configurations, F4 (F4'), F8 and F10/ F14 are respectively the globe minimum state, saddle state and local minimum states. While the initial configurations I8-I11, and I16 are all unstable on the hypersurface, so they transit to the stable final states after relaxation. It seems that the probable transition paths from initial to final states, shown as arrows, are perpendicular to the energy contour line, similar to the typical hypersurface. Based on this principle, we studied the helium pair migration on this energy hypersurface. The reaction coordinates of four migration paths F4-F10, F10-F14, F4-F4' and F8-F10 are plotted and compared with the NEB calculations in Fig. 6.

It's noted that $\mathrm{He}$ atoms are unconstrained to migrate rather than restricted along a certain line during the NEB calculations. Nevertheless, Fig. 6 shows that the shape of the curves, the energy barrier values as well as the transition state positions from the energy hypersurface are all consistent with those from NEB calculations. Therefore, this energy hypersurface should be effective in demonstrating the migration of helium pairs. In the energy hypersurface, F4F4' could be considered as the optimum helium pair migration path with the energy barrier $0.23 \mathrm{eV}$. Besides, the migration path F4-F10-F14 could be regarded as the separation path, i.e., two He atoms are separated from helium pair to far He-He (with the He-He distances changing from $1.58 \AA$ to $4.21 \AA$ ).

It' noted that the actual migration of helium pair in metals is three dimensional. Albeit, this direction [ $\left[\begin{array}{lll}1 & 0 & 0\end{array}\right]$ migration is still meaningful for it is a first step in revealing the migration of helium pair through $\mathrm{He}-$ He energy hypersurface. Different from NEB calculation, this hypersurface map could provide an extensive view of helium pair migration. The transition states of He-He are clearly shown on the map and it's easy to find the optimum migration path.

\section{Conclusion}

From first-principle calculations, we studied the formation and migration of interstitial helium pair in bcc Fe. He-He configurations with $\mathrm{He}-\mathrm{He}$ distance about $1.6 \AA$, called helium pair, exhibit large binding energy. While He-He configurations with He-He distance larger than $2.82 \AA$, called far He-He, exhibit small or negative binding energy. To find out the physics origin of the He-He binding, we put forward a
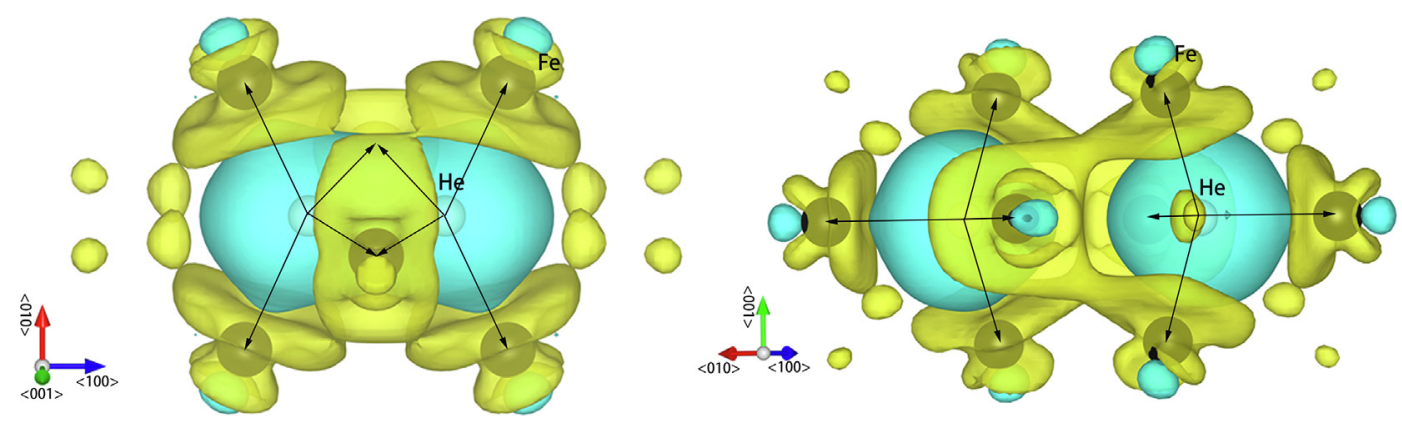

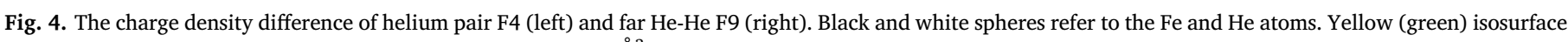

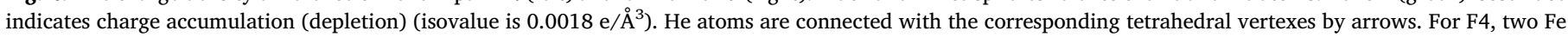

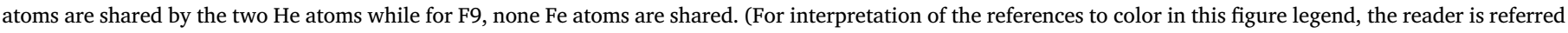
to the web version of this article.) 


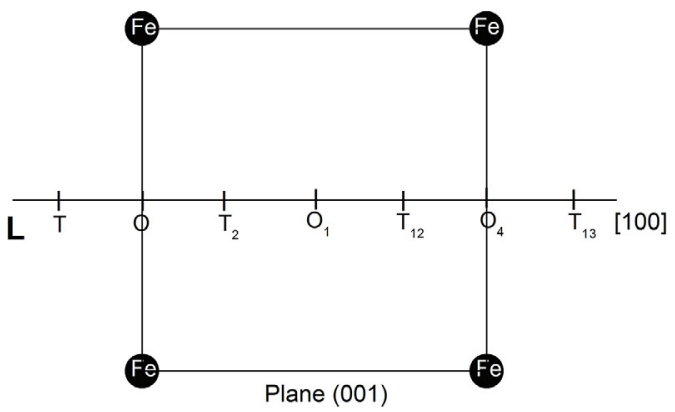

(a)

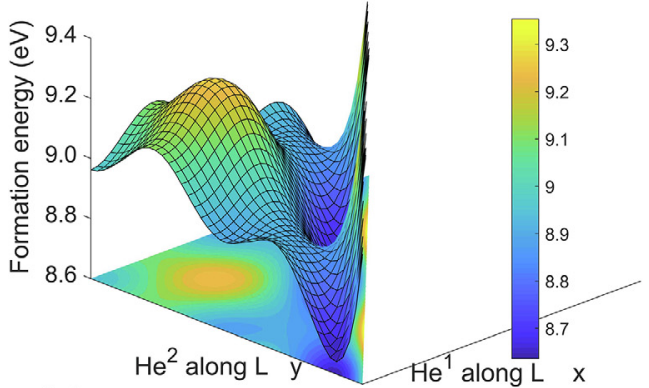

(c)

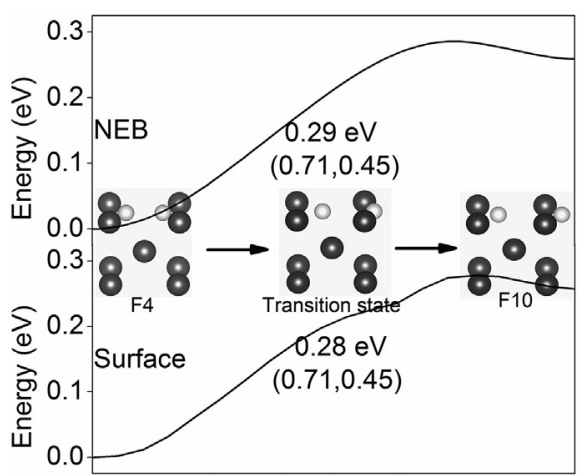

(a)

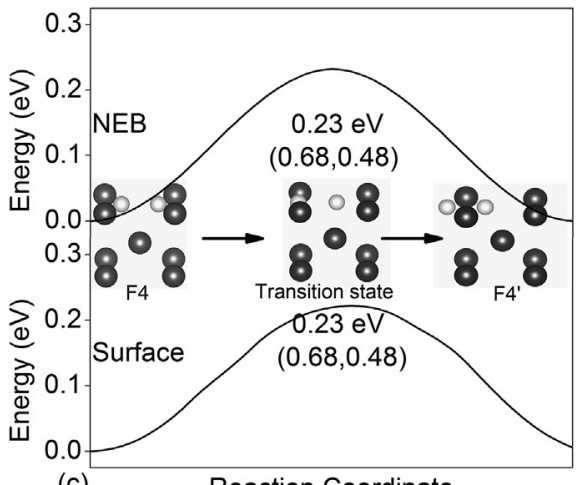

(c)
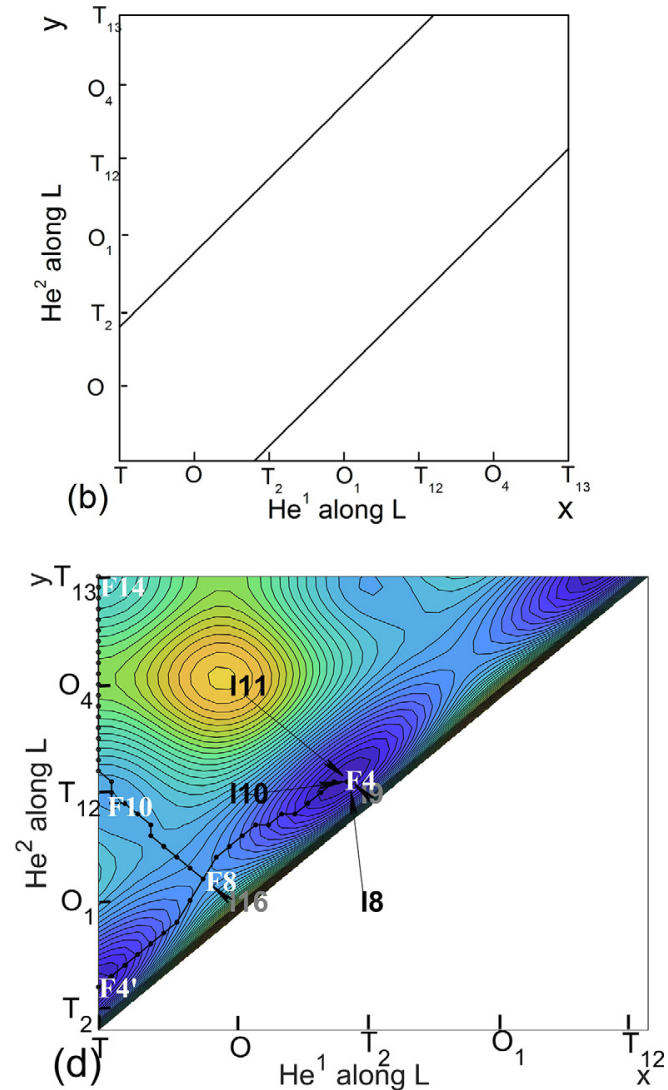

Fig. 5. (a) Line $\mathrm{L}$ in bcc Fe supercell. (b) The $\mathrm{x}-\mathrm{y}$ plane of the He-He energy hypersurface coordinate system. (c) Upper-left triangle part of He-He energy hypersurface. (d) The projection on $x-y$ plane. The migration paths connecting F4, F4', F8, F10 and F14 are also plotted. TIS (T) and OIS (O) are marked along line $\mathrm{L}$ or axes with subscripts consistent with Fig. $1 . \mathrm{He}^{1}$ and $\mathrm{He}^{2}$ refer to the first and second $\mathrm{He}$ atoms of the helium pair. (For interpretation of the references to color in this figure legend, the reader is referred to the web version of this article.)

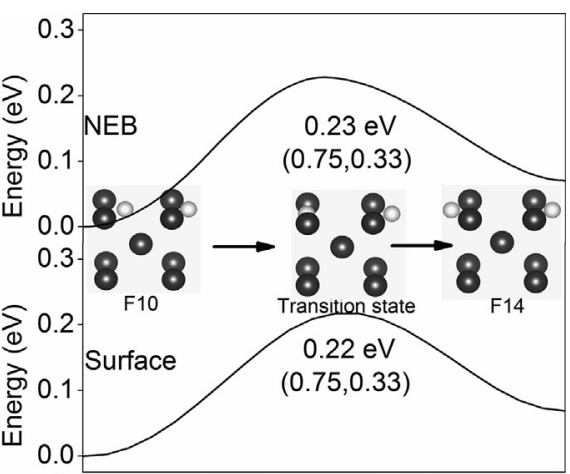

Fig. 6. The reaction coordinates of four migration paths: F4-F10 (a), F10-F14 (b), F4-F4' (c) and F8-F10 (d). In each figure, the upper layer and lower layer show the results from the NEB calculation and the energy hypersurface. The models of the initial, final and transition state are also illustrated; black and white spheres refer to $\mathrm{Fe}$ and $\mathrm{He}$ atoms. The values of the energy barrier and the transition state positions (the coordinates of two helium atoms along direction $\left.\left[\begin{array}{lll}1 & 0 & 0\end{array}\right]\right)$ are also listed correspondingly. formula to decompose the He-He formation energy into two main parts: electron density variation part and lattice distortion part. The results show that electron variation accounts for a major proportion in formation energy. Helium pair could reduce their interface with Fe matrix, so that they cause less electron variation than the far He-He. As a result, two He atoms tend to form helium pair when they get close. It's also found the two He atoms in far He-He are similar with two independent $\mathrm{He}$ atoms. In addition, we construct a He-He energy hypersurface to analyze the migration of helium pair along direction [ $\left.\begin{array}{llll}1 & 0 & 0\end{array}\right]$. The validity of this method was confirmed from NEB calculations. This energy 
hypersurface method could provide an extensive view of helium pair migration paths.

\section{Data availability}

The data required to reproduce these findings are available from the corresponding authors upon reasonable request.

\section{CRediT authorship contribution statement}

Liuliu Li: Conceptualization, Methodology, Investigation, Data curation, Visualization, Formal analysis, Writing - original draft. Jingyi Shi: Software, Writing - review \& editing. Lei Peng: Funding acquisition, Project administration, Resources, Conceptualization, Methodology, Supervision, Writing - review \& editing. Wei Jiang: Supervision, Writing - review \& editing. Guian Qian: Supervision, Writing - review \& editing.

\section{Acknowledgement}

This work is supported by the China National Natural Science Foundation with Grant No. U1730123 and No. 11805131. The numerical calculations in this paper have been done on the supercomputing system in the Supercomputing Center of University of Science and Technology of China.

\section{Appendix A. Supplementary data}

Supplementary data to this article can be found online at https:// doi.org/10.1016/j.commatsci.2019.109192.

\section{References}

[1] H. Trinkaus, B. Singh, Helium accumulation in metals during irradiation-where do we stand? J. Nucl. Mater. 323 (2) (2003) 229-242.

[2] M. Samaras, Multiscale modelling: the role of helium in iron, Mater. Today 12 (11) (2009) 46-53.

[3] P. Trocellier, S. Agarwal, S. Miro, A review on helium mobility in inorganic materials, J. Nucl. Mater. 445 (1) (2014) 128-142.

[4] L. Ventelon, B. Wirth, C. Domain, Helium-self-interstitial atom interaction in $\alpha$ iron, J. Nucl. Mater. 351 (1-3) (2006) 119-132.

[5] X. Gai, T. Lazauskas, R. Smith, S.D. Kenny, Helium bubbles in bcc Fe and their interactions with irradiation, J. Nucl. Mater. 462 (2015) 382-390.

[6] L. Yang, H. Deng, F. Gao, H. Heinisch, R. Kurtz, S. Hu, Y. Li, X. Zu, Atomistic studies of nucleation of He clusters and bubbles in bcc iron, Nucl. Instrum. Meth. Phys. Res., Sect. B 303 (2013) 68-71.

[7] C. Wang, C. Ren, W. Zhang, H. Gong, P. Huai, Z. Zhu, H. Deng, W. Hu, A molecular dynamics study of helium diffusion and clustering in fcc nickel, Comput. Mater. Sci. 107 (2015) 54-57.

[8] P. Zhang, C. Zhang, R. Li, J. Zhao, He-induced vacancy formation in bcc Fe solid from first-principles simulation, J. Nucl. Mater. 444 (1-3) (2014) 147-152.

[9] G. Thomas, W. Swansiger, M. Baskes, Low-temperature helium release in nickel, J. Appl. Phys. 50 (11) (1979) 6942-6947.

[10] G. Thomas, R. Bastasz, Direct evidence for spontaneous precipitation of helium in metals, J. Appl. Phys. 52 (10) (1981) 6426-6428.

[11] A. Haasz, M. Poon, J. Davis, The effect of ion damage on deuterium trapping in tungsten, J. Nucl. Mater. 266 (1999) 520-525.
[12] F.C. Sze, R.P. Doerner, S. Luckhardt, Investigation of plasma exposed W-1\% La2O3 tungsten in a high ion flux, low ion energy, low carbon impurity plasma environment for the International Thermonuclear Experimental Reactor, J. Nucl. Mater. 264 (1-2) (1999) 89-98.

[13] W. Wang, J. Roth, S. Lindig, C. Wu, Blister formation of tungsten due to ion bombardment, J. Nucl. Mater. 299 (2) (2001) 124-131.

[14] W. Wilson, C. Bisson, M. Baskes, Self-trapping of helium in metals, Phys. Rev. B 24 (10) (1981) 5616.

[15] M. Baskes, W. Wilson, Kinetics of helium self-trapping in metals, Phys. Rev. B 27 (4) (1983) 2210

[16] K.O. Henriksson, K. Nordlund, A. Krasheninnikov, J. Keinonen, Difference in formation of hydrogen and helium clusters in tungsten, Appl. Phys. Lett. 87 (16) (2005) 163113.

[17] J. Niu, Q. Zhan, W. Geng, Waltzing of a helium pair in tungsten: migration barrier and trajectory revealed from first-principles, AIP Adv. 4 (6) (2014) 067128.

[18] P. Zhang, T. Zou, J. Zhao, He-He and He-metal interactions in transition metals from first-principles, J. Nucl. Mater. 467 (2015) 465-471.

[19] J. Cao, W. Geng, Migration of helium-pair in metals, J. Nucl. Mater. 478 (2016) 13-25.

[20] C.-C. Fu, F. Willaime, Ab initio study of helium in $\alpha-$ Fe: dissolution, migration, and clustering with vacancies, Phys. Rev. B 72 (6) (2005) 064117.

[21] T. Seletskaia, Y. Osetsky, R.E. Stoller, G.M. Stocks, Magnetic interactions influence the properties of helium defects in iron, Phys. Rev. Lett. 94 (4) (2005) 046403.

[22] C.S. Becquart, C. Domain, Migration energy of He in W revisited by Ab initio calculations, Phys. Rev. Lett. 97 (19) (2006) 196402.

[23] T. Seletskaia, Y. Osetsky, R.E. Stoller, G.M. Stocks, First-principles theory of the energetics of He defects in bcc transition metals, Phys. Rev. B 78 (13) (2008) 134103.

[24] X.T. Zu, L. Yang, F. Gao, S. Peng, H.L. Heinisch, X. Long, R.J. Kurtz, Properties of helium defects in bcc and fcc metals investigated with density functional theory, Phys. Rev. B 80 (5) (2009) 054104.

[25] J. Wang, L.-L. Niu, Y. Zhang, Ab initio study of He-He interactions in homogeneous electron gas, Nucl. Instrum. Meth. Phys. Res., Sect. B 393 (2017) 140-143.

[26] P. Hohenberg, W. Kohn, Inhomogeneous electron gas, Phys. Rev. 136 (3B) (1964) B864.

[27] W. Kohn, L.J. Sham, Self-consistent equations including exchange and correlation effects, Phys. Rev. 140 (4A) (1965) A1133.

[28] G. Kresse, J. Hafner, Ab initio molecular dynamics for liquid metals, Phys. Rev. B 47 (1) (1993) 558

[29] P.E. Blöchl, Projector augmented-wave method, Phys. Rev. B 50 (24) (1994) 17953.

[30] J.P. Perdew, K. Burke, M. Ernzerhof, Generalized gradient approximation made simple, Phys. Rev. Lett. 77 (18) (1996) 3865.

[31] H.J. Monkhorst, J.D. Pack, Special points for Brillouin-zone integrations, Phys. Rev. B 13 (12) (1976) 5188.

[32] Y.-L. Liu, Y. Yu, Z.-H. Dai, Statistical model and first-principles simulation on concentration of HenV cluster and He bubble formation in $\alpha$-Fe and W, J. Nucl. Mater. 456 (2015) 162-173.

[33] G. Henkelman, B.P. Uberuaga, H. Jónsson, A climbing image nudged elastic band method for finding saddle points and minimum energy paths, J. Chem. Phys. 113 (22) (2000) 9901-9904.

[34] R. Li, P. Zhang, X. Li, J. Ding, Y. Wang, J. Zhao, L. Vitos, Effects of Cr and W additions on the stability and migration of He in bcc Fe: a first-principles study, Comput. Mater. Sci. 123 (2016) 85-92.

[35] J. Ding, P. Zhang, D. Sun, Y. Wang, S. Huang, J. Zhao, Energetics of helium-vacancy complexes in Fe-9Cr alloys from first-principles calculations, J. Nucl. Mater. 513 (2019) 143-151.

[36] Y. Bai, J. Shi, L. Peng, X. Wu, L. Li, First principles study on HenV clusters in $\alpha$-Fe bulk and grain boundaries, Comput. Mater. Sci. 139 (2017) 419-429.

[37] P. Zhang, J. Ding, D. Sun, J. Zhao, First-principles study of noble gas atoms in bcc Fe, J. Nucl. Mater. 492 (2017) 134-141.

[38] M. Petersen, S. Wilke, P. Ruggerone, B. Kohler, M. Scheffler, Scattering of rare-gas atoms at a metal surface: evidence of anticorrugation of the helium-atom potential energy surface and the surface electron density, Phys. Rev. Lett. 76 (6) (1996) 995.

[39] J. Ding, P. Zhang, X. Li, Y. Wang, S. Huang, J. Zhao, Magnetism and energetics for vacancy and helium impurity in Fe-9Cr alloy: a first-principles study, Comput. Mater. Sci. 138 (2017) 267-276. 